\title{
Effect of Irbesartan on Oxidative Stress and Serum Inflammatory Factors in Renal Tissues of Type 2 Diabetic Rats
}

\author{
Xingang Zhao', Dongbin Yang2, Weiwei Xu33, Wen Xu4 and Zhengdong $\mathrm{Guo}^{5}$
}

\begin{abstract}
Objective: To determine the effect of irbesartan on the activity of oxidative stress parameters of glutathione (GSH), superoxide enzyme (SOD) and malondialdehyde (MDA), and concentration of serum inflammatory factors of macrophage chemokine-1 (MCP-1), intercellular adhesion molecule-1 (ICAM-1) and tumor necrosis factor alpha (TNF- $\alpha$ ) in the renal tissue of type 2 diabetic rats.

Study Design: An experimental study.

Place and Duration of Study: The First People's Hospital of Ningyang County, Shandong Province, China, from April 2017 to March 2018.

Methodology: Thirty healthy male Sprague Dawley rats were randomly divided into a normal control group, a diabetic model group, and an irbesartan treatment group, 10 in each group. Rat models of type 2 diabetes were prepared by highsugar high-fat diet with low-dose streptozotocin injection. Changes in blood glucose and lipids, serum GSH, SOD, MDA, MCP-1, ICAM-1, and TNF- $\alpha$ levels were determined.

Results: Levels of FBG, TC, TG and LDL, activity of GSH, SOD and MDA, and levels of serum MCP-1, ICAM-1 and TNF- $\alpha$ among three groups were statistically significant (all $p<0.001)$. Compared with the diabetic model group, FBG, TC, TG and LDL levels, MDA activity, MCP-1, ICAM-1 and TNF- $\alpha$ levels all decreased in rats of the irbesartan treatment group (all $p<0.001$ ), and both GSH and SOD activity increased (both $p<0.001$ ).

Conclusion: Irbesartan can improve blood glucose and lipid levels in type 2 diabetic rats, and reduce renal damage by improving oxidative stress and inhibiting the release of inflammatory cytokines.
\end{abstract}

Key Words: Irbesartan, Type 2 diabetes, Rat, Renal tissue, Oxidative stress, Inflammatory factor.

\section{INTRODUCTION}

Continued hyperglycemia not only increases the production of reactive oxygen species (ROS), but also impairs the function of the body's antioxidant system, leading to oxidative stress. This is considered to be one of the important causes of the development and progression of diabetic nephropathy. ${ }^{1}$ Therefore, improving oxidative stress may be an effective measure to prevent and treat diabetic nephropathy. In addition, relevant data also show that some diabetic patients with long-term blood glucose control still suffer from diabetic nephropathy, suggesting that in addition to hyperglycemia, there are some other factors that cause kidney damage in diabetic patients. ${ }^{2,3}$ Therefore, the development of diabetic nephropathy is the result of a combination of multiple factors, yet its exact pathogenesis has not been elucidated.

\footnotetext{
1 Department of Endocrinology / Pathology / Nephrology3 / Geriatrics $^{4}$, The First People's Hospital of Ningyang County, Shandong Province, 271400, China

5 Department of Endocrinology, Affiliated Hospital of Taishan Medical University, 271000, China

Correspondence: Dongbin Yang, Department of Pathology, The First People's Hospital of Ningyang County, Shandong

Province, 271400, China

E-mail:lghuc7@163.com

Received: June 23, 2018; Accepted: November 15, 2018
}

Angiotensin II (Angll) also plays an important role in the occurrence and development of diabetic nephropathy. Inhibiting the production of Angll and interfering with its process is one of the keys to the treatment of diabetic nephropathy. ${ }^{4}$ Irbesartan is a novel Angll receptor antagonist that can completely block the effects of Angll at the receptor level and reduce urine microalbumin, which can prevent the progression of diabetes to some extent. 5

In recent years, more and more evidence shows that chronic inflammation plays a key role in the pathogenesis of diabetic nephropathy. ${ }^{6}$ Therefore, controlling inflammation will also become an important part in the prevention and treatment of diabetic nephropathy. At present, there are few reports on the effects of irbesartan on oxidative stress and serum inflammatory factors in type 2 diabetic rats. In view of this, this experimentinduced type 2 diabetes (T2DM) in normal Sprague Dawley rats with high-fat diet combined with intraperitoneal injection of low-dose streptozotocin. This animal model conformed to the characteristics of human T2DM disease.

The purpose of this study was to determine the effect of irbesartan on the activity of oxidative stress parameters of glutathione (GSH), superoxide enzyme (SOD) and malondialdehyde (MDA), as well as on the concentration of serum inflammatory factors of macrophage chemokine-1 (MCP-1), intercellular adhesion molecule-1 (ICAM-1) 
and tumor necrosis factor alpha $(\mathrm{TNF}-\alpha)$ in the renal tissue of type 2 diabetic rats.

\section{METHODOLOGY}

This study was conducted at The First People's Hospital of Ningyang County, Shandong Province, China, from April 2017 to March 2018, after approval from the Committee on Animal Research and Ethics. The experiment was carried out on 30 healthy male Sprague Dawley rats, which were 7 weeks old and weight $235 \pm$ $15 \mathrm{~g}$, purchased from a commercial laboratory vender. The animal feeded ambient temperature $21.0 \pm 2^{\circ} \mathrm{C}$, humidity $40 \%$ to $70 \%$, divided in cage raising, free food, the natural circadian rhythm of light for a week to adjust the environment and fasted for 16-18 hours. Streptozotocin was dissolved in $0.1 \mathrm{mmol} / \mathrm{L}$ citric acid buffer $(\mathrm{pH}$ $4.5)$ to make $1 \%$ solution. Rats were given a single caudal vein injection of $30 \mathrm{mg} / \mathrm{kg}$ of the above mixed agents, and the high-fat diet ( $40 \%$ fat, $42 \%$ carbohydrate and $18 \%$ protein) was fed on the day of injection. After four weeks of raising, the 24-hour food intake, body weight, blood pressure, non-fasting blood sugar etc. were observed, and insulin inhibition test was performed to evaluate insulin sensitivity. Taking blood sugar $>7.8$ $\mathrm{mmol} / \mathrm{L}$ (taking blood samples under pentobarbital anesthesia) and decreasing insulin sensitivity as criteria for judging a rat model of type 2 diabetes.

After successful replication of diabetic rat model, they were randomly divided into a diabetic model group and an irbesartan treatment group, with 10 in each group, and another 10 rats were placed in the normal control group at the same time. Normal control group was given normal feed, and the diabetic model group and the irbesartan treatment group continued to be given the high-fat diet. Rats in the irbesartan treatment group were given $50 \mathrm{mg} / \mathrm{kg}$ irbesartan. Gavage was given once per day in the morning; the other two groups were given $3 \mathrm{~mL}$ saline, once daily. All three groups were continuously under experiment for 8 weeks. No rat died during the experiment.

At the $8^{\text {th }}$ week of the experiment, fasting blood samples were taken from each group of rats. The fasting blood glucose (FBG), total cholesterol (TC), triglyceride (TG), and low-density lipoprotein (LDL) were determined by an automatic biochemical analyser. The levels of serum inflammatory cytokines MCP-1, ICAM-1 and TNF- $\alpha$ were determined by enzyme-linked immunosorbent assay. After the fasting blood was collected, $3.5 \%$ chloral hydrate was used for intraperitoneal anesthesia. Blood was drawn from the heart. Rats in each group were sacrificed. The right kidney was taken by a pathologist. The residual blood was washed with ice PBS solution. Then $0.5 \mathrm{~g}$ of kidney tissue was taken and added with $4.5 \mathrm{~mL}$ ice physiological saline to make $10 \%$ tissue homo-genate. The supernatant was centrifuged at low temperature, and the activity of GSH, SOD and MDA was measured by chemical colorimetry.

SPSS version 21.0 software was used to process the data. All data was expressed as mean \pm standard deviation. One-way analysis of variance was used for comparison between groups, and SNK test was used for pairwise comparison between groups. The $p<0.05$ was considered statistically significant.

\section{RESULTS}

At the $8^{\text {th }}$ week of the experiment, the differences in the levels of FBG, TC, TG and LDL in the normal control group, the diabetic model group, and the irbesartan treatment group were statistically significant (all $p<0.001$ ). Compared with the diabetic model group, the levels of FBG, TC, TG and LDL in the irbesartan treatment group were significantly lower (all $p<0.001$, Table I).

At the $8^{\text {th }}$ week of the experiment, the differences in the activity of GSH, SOD and MDA in the normal control group, the diabetic model group, and the irbesartan treatment group were statistically significant (all $\mathrm{p}<0.001$ ). Compared with the diabetic model group, the activity of GSH and SOD increased (both $p<0.001$ ) and the activity of MDA decreased in the irbesartan treatment group $(p<0.001$, Table II).

At the $8^{\text {th }}$ week of the experiment, the differences in the levels of inflammatory cytokines MCP-1, ICAM-1 and TNF- $\alpha$ in the normal control group, the diabetic model

Table I: Comparison of the levels of blood glucose and blood lipid among three groups.

\begin{tabular}{l|c|c|c|c}
\hline Parameters & $\begin{array}{c}\text { Normal control } \\
\text { group } \\
(\mathrm{n}=10)\end{array}$ & $\begin{array}{c}\text { Diabetic model } \\
\text { group } \\
(\mathrm{n}=10)\end{array}$ & $\begin{array}{c}\text { Irbesartan } \\
\text { treatment group } \\
(\mathrm{n}=10)\end{array}$ & $\mathrm{p}$-value \\
\hline FBG $(\mathrm{mmol} / \mathrm{L})$ & $7.92 \pm 0.56$ & $26.14 \pm 4.29$ & $19.68 \pm 4.12$ & $<0.001$ \\
$\mathrm{TC}(\mathrm{mmol} / \mathrm{L})$ & $1.03 \pm 0.22$ & $4.16 \pm 0.90$ & $2.49 \pm 1.33$ & $<0.001$ \\
$\mathrm{TG}(\mathrm{mmol} / \mathrm{L})$ & $0.42 \pm 0.05$ & $1.77 \pm 0.68$ & $1.12 \pm 0.35$ & $<0.001$ \\
$\mathrm{LDL}(\mathrm{mmol} / \mathrm{L})$ & $0.14 \pm 0.03$ & $2.61 \pm 0.89$ & $0.96 \pm 0.54$ & $<0.001$ \\
\hline
\end{tabular}

Table II: Comparison of the activity of GSH, SOD and MDA in the renal tissue among three groups.

\begin{tabular}{l|c|c|c|c}
\hline Parameters & $\begin{array}{c}\text { Normal control } \\
\text { group } \\
(\mathrm{n}=10)\end{array}$ & $\begin{array}{c}\text { Diabetic model } \\
\text { group } \\
(\mathrm{n}=10)\end{array}$ & $\begin{array}{c}\text { Irbesartan } \\
\text { treatment group } \\
(\mathrm{n}=10)\end{array}$ & $\mathrm{p}$-value \\
\hline GSH (mmol/L) & $3.81 \pm 1.31$ & $2.13 \pm 0.05$ & $3.35 \pm 0.16$ & $<0.001$ \\
$\mathrm{SOD}(\mathrm{mmol} / \mathrm{L})$ & $375.62 \pm 33.24$ & $204.78 \pm 27.05$ & $284.97 \pm 42.35$ & $<0.001$ \\
$\mathrm{MDA}(\mathrm{mmol} / \mathrm{L})$ & $19.95 \pm 7.14$ & $64.36 \pm 17.96$ & $34.84 \pm 9.19$ & $<0.001$ \\
\hline
\end{tabular}

Table III: Comparison of the levels of serum inflammatory factors among the three groups.

\begin{tabular}{l|c|c|c|c}
\hline Parameters & $\begin{array}{c}\text { Normal control } \\
\text { group } \\
(\mathrm{n}=10)\end{array}$ & $\begin{array}{c}\text { Diabetic model } \\
\text { group } \\
(\mathrm{n}=10)\end{array}$ & $\begin{array}{c}\text { Irbesartan } \\
\text { treatment group } \\
(\mathrm{n}=10)\end{array}$ & p-value \\
\hline MCP-1 (mmol/L) & $8.96 \pm 1.10$ & $15.02 \pm 1.58$ & $11.06 \pm 1.23$ & $<0.001$ \\
ICAM-1 (mmol/L) & $21.45 \pm 2.72$ & $33.61 \pm 4.29$ & $26.38 \pm 3.43$ & $<0.001$ \\
TNF- $\alpha(\mathrm{mmol} / \mathrm{L})$ & $10.95 \pm 2.02$ & $23.36 \pm 4.49$ & $15.15 \pm 2.78$ & $<0.001$ \\
\hline
\end{tabular}


group, and the irbesartan treatment group were statistically significant (all $p<0.001$ ). Compared with the diabetic model group, the levels of serum MCP-1, ICAM1 and TNF- $\alpha$ decreased in the irbesartan treatment group (all $p<0.001$, Table III).

\section{DISCUSSION}

The occurrence of diabetic nephropathy is closely related to hyperglycemia, and the progress of diabetic nephropathy can be delayed due to strict control of blood glucose.7,8 Due to impaired biological regulation of insulin, diabetic patients are often accompanied by disorders of lipid metabolism, which is even more evident in patients with diabetic nephropathy. Disorders of lipid metabolism are associated with blood stasis in the renal microcirculation and are an independent risk factor for the development of diabetic nephropathy. ${ }^{9}$ The occurrence of diabetic nephropathy is associated with lipid alterations mainly manifested as elevated TG, and this change is present early in diabetic nephropathy. 10 Studies have found that elevated TG and decreased HDL-C in patients with diabetic nephropathy are significantly associated with changes in micro-albuminuria. This change in lipids affects the development of diabetic nephropathy. ${ }^{11}$ This study showed that, compared with the diabetic model group, the levels of FBG, TC, TG and LDL in the irbesartan treatment group were lower, indicating that irbesartan can reduce blood glucose and lipid levels in type 2 diabetic rats, and it can regulate blood glucose and lipid disorders. Irbesartan can regulate blood lipid while reducing blood sugar. The mechanism may be that fat cells contain renin angiotensin - aldosterone system active ingredient, and irbesartan as its antagonist, can adjust the lipids by inhibiting the storage activity of fat cells. ${ }^{12}$

When diabetic nephropathy occurs, the body is often in an oxidative stress state and is prone to produce excessive oxygen-free radicals. When the generation of reactive oxygen species increases and cannot be completely eliminated, some unstable molecules in the cell (such as lipids, proteins, DNA, etc.) are easily oxidised, causing their structural change and dysfunction. MDA is one of the indicators reflecting the lipid peroxidation of oxygen radicals, which is produced in the kidney intrinsically or by circulating inflammatory cells. ${ }^{13}$ MDA content in the kidneys of DM rats can be detected to reflect the status of renal oxidative stress. SOD has the ability to clear a variety of oxygen-free radicals, and detection of its changes can indirectly reflect the body's antioxidant capacity. ${ }^{14} \mathrm{GSH}$ is a tripeptide that is ubiquitous in the cytoplasm and accounts for $90 \%$ of intracellular non-protein thiols and protects cells from oxidative damage. At the same time, GSH also serves as a substrate for GSH-Px to assist GSH-Px in scavenging-free radicals. ${ }^{15}$ This study showed that, compared with the diabetic model group, the activity of
GSH and SOD increased and the MDA activity decreased in the irbesartan treatment group, revealing that irbesartan can reduce the level of oxidative stress in the renal tissue of type 2 diabetic rats by increasing GSH and SOD, and reducing MDA in renal tissue, thus exerting a protective effect on kidneys.

More and more evidence show that macrophage infiltration and overexpression of inflammatory factors such as cytokines, chemokines, and adhesion molecules are closely related to the development of diabetic nephropathy. ${ }^{16}$ Monocyte chemoattractant protein-1 (MCP-1), the strongest monocyte chemotactic factor, is mainly secreted by monocytes and macrophages, but in some cases, it can also be secreted by renal tubular epithelial cells and mesangial cells. ${ }^{17}$ Clinical and experimental studies have found that MCP-1 is upregulated in diabetic nephropathy, and urine or blood levels of MCP-1 are associated with macrophage recruitment, proteinuria, renal interstitial injury, and disease progression. 18 The role of intercellular adhesion molecule-1 (ICAM-1) in the pathogenesis of diabetic nephropathy is increasingly valued. Studies have found that ICAM-1 mediated monocyte-macrophage adhesion to glomerular vascular endothelial cells plays an important role in the pathophysiology of diabetic nephropathy and can accelerate the progression of diabetic nephropathy. ${ }^{19,20}$ Tumor necrosis factor alpha (TNF- $\alpha$ ) is a pro-inflammatory factor that plays an important role in the process of inflammation. In the diabetic state, high glucose and hemodynamic changes can stimulate the release of TNF- $\alpha$. TNF- $\alpha$ can induce reactive oxygen species (ROS), oxidative stress, and the expression of MCP-1 and ICAM-1. This study showed that, compared with the diabetic model group, the levels of MCP-1, ICAM-1 and TNF- $\alpha$ were lower in the irbesartan treatment group, suggesting that irbesartan can decrease the secondary inflammatory response and thus reduce the damage of renal tissue.

\section{CONCLUSION}

Irbesartan can improve blood glucose and lipid levels in type 2 diabetic rats, and reduce renal damage by improving oxidative stress and inhibiting the release of inflammatory cytokines.

\section{REFERENCES}

1. Hou Y, Shi Y, Han B, Liu X, Qiao X, Qi Y, et al. The antioxidant peptide SS31 prevents oxidative stress, downregulates CD36 and improves renal function in diabetic nephropathy. Nephrol Dial Transplant 2018; 33:1908-18.

2. Moumen A, Bouziane A, Meftah A, Errahali $Y$, Eljadi $H$, Elmoussaoui $\mathrm{S}$, et al. Chronic kidney failure and carotid atherosclerosis in diabetic patient. Presse Med 2016; 45:e341-9.

3. Arif M, Arif MK, Arif MS. An evaluation of renal biopsy in type-II diabetic patients. J Coll Physicians Surg Pak 2009; 19:627-31. 
4. Batlle D, Wysocki J, Soler MJ, Ranganath K. Angiotensinconverting enzyme 2: Enhancing the degradation of angiotensin ii as a potential therapy for diabetic nephropathy. Kidney Int 2012; 81:520-8.

5. Mohamed EA, Ahmed HI, Zaky HS. Protective effect of irbesartan against doxorubicin-induced nephrotoxicity in rats: Implication of AMPK, PI3K/Akt, and mTOR signaling pathways. Can J Physiol Pharmacol 2018; 96:1209-17.

6. Abrahamian $\mathrm{H}$, Endler $\mathrm{G}$, Exner M, Mauler H, Raith $\mathrm{M}$, Endler L, et al. Association of low-grade inflammation with nephropathy in type 2 diabetic patients: Role of elevated CRP-levels and 2 different gene-polymorphisms of proinflammatory cytokines. Exp Clin Endocrinol Diabetes 2007; 115:38-41.

7. Al Hroob AM, Abukhalil MH, Alghonmeen RD, Mahmoud AM. Ginger alleviates hyperglycemia-induced oxidative stress, inflammation and apoptosis and protects rats against diabetic nephropathy. Biomed Pharmacother 2018; 106:381-9.

8. Mundinger TO, Cooper E, Coleman MP, Taborsky GJ Jr. Shortterm diabetic hyperglycemia suppresses celiac ganglia neurotransmission, thereby impairing sympathetically mediated glucagon responses. Am J Physiol Endocrinol Metab 2015; 309:E246-55.

9. Liu N, Yuan W, Zhang X, Liu X, Jia F. Effects of genistein on metabolism disorder of diabetic mellitus rats and their kidney pathology. Chin J Compar Med 2005; 15:200-2.

10. Kawanami D, Matoba K, Utsunomiya K. Dyslipidemia in diabetic nephropathy. Renal Replacement Therapy 2016; 2:16-25.

11. Sun X, Xiao Y, Li PM, Ma XY, Sun XJ, Lv WS, et al. Association of serum high-density lipoprotein cholesterol with microalbuminuria in type 2 diabetes patients. Lipids Health Dis 2018; 17:229.

12. Svishchenko lep, Kotsiuruba AV, Mehed OF, Bukhanevich OM, Radchenko VV, Hula NM. Effect of irbesartan--angiotensin II type I receptor inhibitor on oxidative metabolism of lipids in essential hyper-tension. Fiziol Zh 2002; 48:31-6.
13. Utsumi K, Yasuda F, Watanabe Y, Higo S, Hirama A, Fujita E, et al. Effects of olmesartan and imidapril on the plasma adiponectin, P-selectin, and MDA-LDL levels of diabetic nephropathy patients. Clinica Chimica Acta 2012; 413:348-9.

14. Dominko K, Dikic D, Hecimovic S. Enhanced activity of superoxide dismutase is a common response to dietary and genetically induced increased cholesterol levels. Nutr Neurosci 2018:1-13.

15. Look MP, Rockstroh JK, Rao GS, Kreuzer KA, Barton S, Lemoch $\mathrm{H}$, et al. Serum selenium, plasma glutathione (GSH) and erythrocyte glutathione peroxidase (GSH-Px)-levels in asymptomatic versus symptomatic human immunodeficiency virus-1 (HIV-1)-infection. Eur J Clin Nutr 1997; 51:266-72.

16. Navarro-González JF, Mora-Fernández $\mathrm{C}$. The role of inflammatory cytokines in diabetic nephropathy. J Am Soc Nephrol 2008; 19:433-42.

17. Katakami N, Matsuhisa M, Kaneto H, Matsuoka TA, Imamura K, Ishibashi F, et al. Monocyte chemoattractant protein-1 (MCP-1) gene polymorphism as a potential risk factor for diabetic retinopathy in japanese patients with type 2 diabetes. Diabetes Res Clin Pract 2010; 89:e9-12.

18. Tilak P, Khashim Z, Kumpatla S, Babu M, Viswanathan V. Clinical significance of urinary monocyte chemoattractant protein-1 (UMCP-1) in Indian type 2 diabetic patients at different stages of diabetic nephropathy. Int J Diabetes Mellitus 2010; 2:15-9.

19. Chow FY, Nikolic-Paterson DJ, Ozols E, Atkins RC, Tesch GH. Intercellular adhesion molecule-1 deficiency is protective against nephropathy in type 2 diabetic $\mathrm{db} / \mathrm{db}$ mice. J Am Soc Nephrol 2005; 16:1711-22.

20. Kamiuchi K, Hasegawa G, Obayashi H, Kitamura A, Ishii M, Yano $\mathrm{M}$, et al. Intercellular adhesion molecule-1 (ICAM-1) polymorphism is associated with diabetic retinopathy in type 2 diabetes mellitus. Diabet Med 2002; 19:371-6. 